\title{
Sinais e sintomas relacionados à baixa acuidade visual em escolares do nordeste do Rio Grande do Sul.
}

Signs and symptoms related to low visual acuity in schoolchildren from the Northeast of Rio Grande do Sul.

\author{
Ellen Zatti Ramos Simionato* \\ Jonathan Soldera** \\ Eduardo Machado Estevão Pires** \\ Filipe Rech Bassani*** \\ Eduardo Schmidt Rizzon**** \\ Gustavo Basso Poleto*****
}

\section{Resumo}

O objetivo do estudo foi associar os principais sinais e sintomas oculares da infância com a baixa acuidade visual em escolares do nordeste do Rio Grande do Sul. Durante o Projeto Saúde é Cidadania/Ação Comunitária, do período de março a setembro de 2006, foi realizado - com 338 escolares de quatro a 15 anos, os quais chegaram ao serviço de forma livre e espontânea - o teste de acuidade visual com a tabela de Snellen e um questionário indagando: a presença de dificuldade para ver o quadro; cefaléia; dor; vermelhidão ou prurido ocular. Foi considerado parâmetro para a baixa acuidade visual aquela menor ou igual a 20/20 em ambos os olhos. A estatística foi realizada pelo teste do qui-quadrado e o cálculo da sensibilidade (s), especificidade (e), valor preditivo positivo (vpp) e likelihood ratio $+(\mathrm{lr}+)$. Foi estatisticamente significativa a relação entre a baixa acuidade visual com a dificuldade para enxergar o quadro na escola $(\mathrm{p}<0,001, \mathrm{~s}=26 \%$, $\mathrm{e}=93 \%, \mathrm{vpp}=51 \%, \mathrm{lr}+=3,7)$ e com a dor ocular $(\mathrm{p}=0,002, \mathrm{~s}=23 \%, \mathrm{e}=90 \%, \mathrm{vpp}=38 \%, \mathrm{l}+=2,3)$. Não foi estatisticamente significativa a relação entre a baixa acuidade visual e a cefaléia $(\mathrm{p}=0,3, \mathrm{~s}=26 \%, \mathrm{e}=79 \%, \mathrm{pp}=24 \%, \mathrm{lr}+=1,2)$, prurido $\operatorname{ocular}(\mathrm{p}=0,06, \mathrm{~s}=26 \%, \mathrm{e}=83 \%, \mathrm{vpp}=28 \%, \mathrm{lr}+=1,5)$ e vermelhidão ocular $(\mathrm{p}=0,8, \mathrm{~s}=11 \%, \mathrm{e}=88 \%, \mathrm{vpp}=21 \%, \mathrm{l}+=0,9)$. A relação da presença de pelo menos uma dessas queixas com a baixa acuidade visual foi significativa $(p=0,004, s=58 \%$, $\mathrm{e}=60 \%, \mathrm{vpp}=27 \%, \mathrm{lr}+=1,4)$.

Crianças em idade escolar com dificuldade em enxergar o quadro ou dor ocular têm uma probabilidade maior de possuírem baixa acuidade visual, enquanto crianças com cefaléia, vermelhidão ou prurido ocular não fazem parte dessa associação.

Palavras-chave: Acuidade visual; Saúde Escolar;

Manifestações Oculares.
Key Words: Family Practice; Gastrointestinal

Diseases; Primary Health Care.

\footnotetext{
* Médica especialista em Oftalmologia Pediátrica. Ambulatório Central da Universidade de Caxias do Sul. Caxias do Sul, Rio Grande do Sul, Brasil **Aluno, Monitor da Área de Oftalmologia do Projeto Saúde É Cidadania/Ação Comunitária, Faculdade de Medicina, Universidade de Caxias do Sul, Caxias do Sul, Rio Grande do Sul, Brasil.

***Aluno, Bolsista de Iniciação Profissional da Área de Oftalmologia do Projeto Saúde É Cidadania/Ação Comunitária, Faculdade de Medicina, Universidade de Caxias do Sul, Caxias do Sul, Rio Grande do Sul, Brasil.

****Médico, Bolsista de Iniciação Profissional da Área de Oftalmologia do Projeto Saúde É Cidadania/Ação Comunitária, Faculdade de Medicina, Universidade de Caxias do Sul, Caxias do Sul, Rio Grande do Sul, Brasil.

*****Aluno, Faculdade de Medicina, Universidade de Caxias do Sul. Voluntário da Área de Oftalmologia do Projeto Saúde É Cidadania/Ação Comunitária, Caxias do Sul, Rio Grande do Sul, Brasil.
} 
Abstract

Purpose: Associate the most important eye symptoms and signs of the childhood to low visual acuity in children from the northeast of Rio Grande do Sul. Methods: During the Project "Health and the Citizen/Community Action", carried out from March to September 2006, a visual acuity test was performed in 338 schoolchildren between 4 and 15 years of age, who looked for the service spontaneously. The screening was performed using the Snellen chart and a questionnaire asking about difficulty to see the blackboard at school, beadache, eye redness, pain or itch. Acuity less or equal to 20/20 in both eyes was used as parameter for low visual acuity. Statistical analysis was performed using the chi-square test and sensitivity (se), specificity (sp), positive predictive value ( $p p v)$ and likelihood ratio + (lr+). Results: There was a statistically significant relationship between low visual acuity and difficulty to see the blackboard at school $(p<0.001$, se $=26 \%$, $s p=93 \%, p p v=51 \%, l r+=3.7)$ and eye $p a i n(p=0.002, s e=23 \%$, $s p=90 \%, p p v=38 \%, l r+=2.3)$. No statistically significant association was found between low visual acuity and headache $(p=0.3, s e=26 \%$, $s p=79 \%, p p v=24 \%, l r+=1.2)$, eye itch ( $p=0.06, s e=26 \%$, $s p=83 \%, p p v=28 \%, l r+=1.5)$ and eye redness $(p=0.8, s e=11 \%$, $s p=88 \%, p p v=21 \%, l r+=0.9)$. On the other hand, there was a statistically significant relationship between the presence of at least one of these conditions and low visual acuity $(p=0.004$, se $=58 \%$, $s p=60 \%, p p v=27 \%, \mathrm{lr}+=1.4)$. Conclusion: Children at school age with difficulty to see the blackboard or with eye pain are more subject to low visual acuity, while no such association was found in children complaining from headache, eye pain or eye redness.

\section{Introdução}

É de grande repercussão a baixa acuidade visual sobre o desenvolvimento de uma criança. Por isso, muitos autores reforçam a necessidade de triagem de acuidade visual, a fim de se detectar alterações visuais precocemente em crianças em idade escolar ${ }^{1,2,3,4}$.

Deve-se levar em consideração que é no período escolar que as crianças começam a necessitar de grande esforço ocular, sendo nessa faixa etária que distúrbios préexistentes podem vir a se manifestar. As principais conseqüências disso são: baixo rendimento escolar, distúrbios psicológicos e emocionais, além de alterações no desenvolvimento da personalidade ${ }^{5,6,7}$. Um fato preocupante é que muitos professores não possuem conhecimento suficiente quanto à saúde ocular e, por esse motivo, não conseguem identificar a baixa acuidade visual e encaminhar as crianças a um profissional da área ${ }^{8}$. Diversos sinais e sintomas, como dificuldade de ver o quadro, cefaléia, dor, vermelhidão e prurido ocular, que não recebem a devida importância pelos pais e professores, podem significar diminuição da acuidade visual. Cerca de $20 \%$ a $25 \%$ das crianças em idade escolar apresentam algum tipo de problema ocular ${ }^{5,6}$, cuja detecção precoce é essencial para a minimização ou eliminação de suas conseqüências.

É recomendado que o exame de acuidade visual seja realizado pelo pediatra ou médico de família da criança, o qual jamais deve considerar que a criança está sendo propriamente examinada fora de seu consultório ${ }^{9,10}$. Embora exista certa controvérsia quanto a quem deve realizar esse exame - seja o oftalmologista, quando da entrada da criança na escola, o pediatra ou o médico de família, quando a criança tem de quatro a sete anos, ou o enfermeiro ou o professor da escola -, a não-realização dele pelo pediatra ou médico de família que acompanha o crescimento e desenvolvimento da criança pode levar ao litígio por um diagnóstico tardio de ambliopia ou retinoblastoma ${ }^{11}$.

O teste de acuidade visual tem por objetivo detectar precocemente deficiências como ambliopia e erros de refração ${ }^{5,6}$, não exigindo alto nível de especialização do examinador nem demandando alto custo. Tal teste tem uma acurácia de $87,1 \%{ }^{6}$, sendo a triagem da acuidade visual perfeitamente viável e de fácil execução, podendo ser aplicada por professores e enfermeiros. $\mathrm{O}$ método mais usado é a tabela de Snellen, criada por Hermann Snellen, um oftalmologista francês, no século XIX. O método baseia-se na letra $\mathrm{E}$ voltada nas quatro direções e organizadas em linhas ${ }^{12}$.

\section{Métodos}

O presente estudo analisou crianças em idade escolar, de 4 a 15 anos, no serviço de Oftalmologia do 
Projeto Saúde é Cidadania/Ação Comunitária, que foi desenvolvido pela Pró-Reitoria de Extensão por meio da Coordenadoria de Desenvolvimento Social da Universidade de Caxias do Sul (UCS).

Nesse projeto, alunos e professores da UCS visitaram cidades e bairros do nordeste do Rio Grande do Sul que apresentam dificuldades de acesso ao atendimento de saúde primário. Foram realizados diversos exames de triagem, como medida da glicose capilar, toque retal, aferição da pressão arterial, exame dermatoscópio de lesões cutâneas, medida da acuidade visual, dentre outros. Foram atendidos gratuitamente, nessa oportunidade, todos os pacientes que buscaram o serviço, sendo a demanda espontânea.

A aferição da acuidade visual foi feita com a tabela de Snellen com optotipos E. Para a realização dos testes foram escolhidos lugares tranqüilos e com iluminação adequada. As tabelas foram fixadas a uma distância de 5 metros do examinado, e a linha correspondente à acuidade visual 20/20 foi colocada ao nível dos olhos do mesmo. Antes do início do teste, foi esclarecido a cada um o objetivo e o método do teste, a fim de facilitar a compreensão e a identificação dos optotipos da tabela. Foi apresentado o optotipo E em suas variações, para que o sujeito pudesse indicar a direção do optotipo. Essa explicação foi realizada com a pessoa situada próxima à tabela. Para facilitar a compreensão e a resposta, foi dada ao sujeito uma régua em formato de E, a qual ele podia girar conforme a posição da letra na tabela.

A acuidade visual foi aferida em cada olho separadamente, primeiro no direito e, a seguir, no esquerdo. Iniciou-se apontando para o primeiro optotipo da tabela, continuando de acordo com as linhas horizontais, de cima para baixo. Quando o sujeito apresentou dificuldade em determinada etapa, solicitou-se que ele repetisse a linha anterior. Foi registrada a acuidade visual da linha em que o examinado acertou pelo menos $70 \%$ dos optotipos sem apresentar dificuldades, sendo utilizada a classificação fracionada para a tomada de notas. Esse exame foi realizado pelos acadêmicos do $10^{\circ}$ e $12^{\circ}$ semestre de Medicina após treinamento e sob a supervisão dos orientadores.

O exame foi realizado apenas com o consentimento dos responsáveis pelo sujeito, sendo preenchido um Termo de Consentimento Livre e Esclarecido, o qual foi aprovado pelo comitê de Ética em Pesquisa da Universidade de Caxias do Sul, conforme os preceitos da ética em pespesquisa em seres humanos.

Ao término do exame, os sujeitos, com auxílio de seus responsáveis, responderam a um questionário que indagava sobre a idade, sexo e a presença dos sinais e sintomas levantados pelo grupo pesquisador: dificuldade de ver o quadro, cefaléia, dor, vermelhidão e prurido ocular.

Foram incluídos no trabalho todos os sujeitos de 4 a 15 anos que buscaram o serviço de forma livre e espontânea, sendo excluídos os portadores de deficiência considerados incapazes de, satisfatoriamente, responderem ao exame com a tabela de Snellen. Foi considerada como baixa acuidade visual aquela inferior a 20/20 em ambos os olhos. Isso para fins de análise estatística, já que se sabe que em crianças de 4 a 7 anos pode-se usar como parâmetro a acuidade 20/25, dado o fato de que a visão de crianças nessa faixa etária ainda se encontra em desenvolvimento.

A análise estatística foi realizada na plataforma estatística SPSS for Windows 14.0, utilizando-se o teste do qui-quadrado para calcular a associação entre os cinco sinais e sintomas citados e a baixa acuidade visual. Na plataforma Pepi4, foram calculadas prevalências com intervalo de confiança 95\% por meio do Teste de Fisher. Foram calculados os valores da sensibilidade, especificidade, acurácia, valor preditivo positivo (VPP) e negativo (VPN) e likelihood ratio $+(\mathrm{LR}+)$ para cada um dos sinais e sintomas e associações.

\section{Resultados}

Das 338 crianças incluídas no estudo, 167 (49,4\%, IC95\%: 44\%-54,7\%) eram meninos e 171 (50,6\%, IC95\%: 45,2\%-55,9\%), meninas. Em relação à faixa etária, 66 (19,5\%, IC95\%: 15,5\%-24\%) tinham de 4 a 7 anos, 169 (50\%, IC95\%: 44,6\%-55,3\%) tinham de 8 a 11 anos e 103 (30,5\%, IC95\%: 25,7\%-35,5\%) tinham de 12 a 15 anos. 
Tabela 1. Prevalência dos sinais e sintomas pesquisados na amostra.

\begin{tabular}{lccc}
\hline \multicolumn{1}{c}{ Sinais e sintomas } & N & Prevalência & IC 95\% \\
\hline Dificuldade para enxergar o quadro & 35 & $10,3 \%$ & $7,4 \%-13,9 \%$ \\
Cefaléia & 74 & $21,8 \%$ & $17,7 \%-26,5 \%$ \\
Dor ocular & 42 & $12,4 \%$ & $9,2 \%-16,2 \%$ \\
Vermelhidão ocular & 38 & $11,2 \%$ & $8,1 \%-14,9 \%$ \\
Prurido ocular & 63 & $18,6 \%$ & $14,7 \%-23,0 \%$ \\
Dificuldade para enxergar o quadro ou dor ocular & 68 & $20,1 \%$ & $16,1 \%-24,6 \%$ \\
Qualquer um & 146 & $43,1 \%$ & $37,9 \%-48,5 \%$ \\
\hline
\end{tabular}

Tabela 2. Relação dos sinais e sintomas pesquisados com a baixa acuidade visual.

\begin{tabular}{lccc}
\hline \multicolumn{1}{c}{ Sinais e sintomas } & $\begin{array}{c}\text { \% dos pacientes com } \\
\text { acuidade visual normal }\end{array}$ & Odds ratio (IC 95\%) & P \\
\hline Dificuldade para enxergar o quadro & $6,6 \%$ & $5,35(2,5-11,1)$ & $<0,001$ \\
Cefaléia & $20,7 \%$ & $1,37(0,7-2,5)$ & 0,3 \\
Dor ocular & $9,6 \%$ & $2,88(1,4-5,7)$ & 0,002 \\
Vermelhidão ocular & $11,1 \%$ & $1,06(0,4-2,4)$ & 0,87 \\
Prurido ocular & $16,6 \%$ & $1,8(0,9-3,3)$ & 0,064 \\
Dificuldade para enxergar o quadro ou dor ocular & $14,8 \%$ & $4,02(2,2-7,2)$ & $<0,001$ \\
Qualquer um & $39,2 \%$ & $2,21(1,2-3,7)$ & 0,004 \\
\hline
\end{tabular}

Encontrou-se, nessa amostra, baixa acuidade visual em 68 crianças $(20,1 \%$, IC95\%: 16,1\%-24,6\%). A prevalência dos sinais e sintomas pesquisados pode ser visualizada na Tabela 1.

Houve associação entre a baixa acuidade visual com a dificuldade para enxergar o quadro e a dor ocular, conforme descrito na Tabela 2 .

Os cálculos de sensibilidade, especificidade, acurácia, VPP, VPN e LR + podem ser observados de acordo com a Tabela 3.

\section{Discussão}

O estudo demonstrou que a baixa acuidade visual em escolares do nordeste do Rio Grande do Sul possui uma relação significativamente estatística com os sinais e sintomas investigados.

Também foi quantificada, no presente estudo, a prevalência de baixa acuidade visual nos pacientes atendidos, que foi de 20,1\%, um valor aproximado aos valores de outros estudos brasileiros, como: 19\% em Porto Alegre ${ }^{13}$, 28,5\% em Jundiaí ${ }^{14}$, 22,4\% em São Paulo ${ }^{15}$, 17,8\% em Londrina $^{16}$ e $11,9 \%$ em São Carlos ${ }^{17}$.

$\mathrm{Na}$ amostra estudada, obteve-se um total de 35 crianças com a queixa de dificuldade para enxergar o quadro, estando a mesma presente em $26,4 \%$ dos pacientes com baixa acuidade visual $(p<0,001)$. Erros de refração são causas comuns de dificuldade visual infantil e podem cursar com baixa acuidade visual para longe, o que pode causar dificuldade para a criança enxergar o quadro na escola, que normalmente se encontra distante e mais alto do que o local onde a criança está sentada. Deve-se notar, porém, que, embora esse sintoma seja específico, é pouco sensível. Crianças com essa queixa devem ser submetidas à avaliação oftalmológica completa, enquanto sua ausência 
Tabela 3. Análise estatística da presença dos sinais e sintomas na baixa acuidade visual.

\begin{tabular}{|c|c|c|c|c|c|c|}
\hline Sinais e sintomas & Sensibilidade & Especificidade & Acurácia & VPP & VPN & LR+ \\
\hline $\begin{array}{l}\text { Dificuldade para enxergar o } \\
\text { quadro }\end{array}$ & $26 \%$ & $93 \%$ & $80 \%$ & $51 \%$ & $83 \%$ & 3,7 \\
\hline Cefaléia & $26 \%$ & $79 \%$ & $68 \%$ & $24 \%$ & $81 \%$ & 1,2 \\
\hline Dor ocular & $23 \%$ & $90 \%$ & $76 \%$ & $38 \%$ & $82 \%$ & 2,3 \\
\hline Vermelhidão ocular & $11 \%$ & $88 \%$ & $73 \%$ & $21 \%$ & $80 \%$ & 0,9 \\
\hline Prurido ocular & $26 \%$ & $83 \%$ & $71 \%$ & $28 \%$ & $81 \%$ & 1,5 \\
\hline $\begin{array}{l}\text { Dificuldade para enxergar o } \\
\text { quadro ou dor ocular }\end{array}$ & $41 \%$ & $85 \%$ & $76 \%$ & $41 \%$ & $85 \%$ & 2,7 \\
\hline Qualquer um & $28 \%$ & $60 \%$ & $60 \%$ & $27 \%$ & $85 \%$ & 1,4 \\
\hline
\end{tabular}

não deve significar a ausência de deficiência visual, sendo indicado o exame de triagem visual habitual.

Dor ocular foi um sintoma referido por 42 crianças entrevistadas, sendo encontrado em $23,5 \%$ das crianças com baixa acuidade visual $(p=0,002)$. Esse sintoma, geralmente associado a doenças que não afetam a acuidade visual, pode estar presente em pacientes com erros de refração, já que estes necessitam muitas vezes de grande esforço em ambiente escolar ou domiciliar para visualizar objetos que se encontram distantes deles, o que pode eventualmente causar esse sintoma. A presença desse sintoma deve levar a uma avaliação oftalmológica completa, para excluir doenças oftalmológicas da infância que cursam com ele e que não afetam a acuidade visual, além de já ser possível a investigação de erros de refração. Sua ausência, entretanto, não isenta a criança de ser submetida à triagem visual habitual.

Foi observado um total de 74 crianças queixandose de cefaléia, estando presente em $26,4 \%$ das crianças com baixa acuidade visual $(p=0,3)$. O estudo holandês de Hendricks et al. encontrou uma pequena, porém, estatisticamente significativa relação entre erros de refração e a cefaléia em crianças, concluindo que erros de refração podem ser um fator de risco para cefaléia em crianças ${ }^{18}$. No presente estudo, porém, esse achado não teve relação com a baixa acuidade visual e não foi suficientemente sensível nem específico. Embora a cefaléia apresente uma relação, na cultura popular, com a baixa acuidade visual, o encaminhamento de crianças apresentando esse sintoma para avaliação oftalmológica completa compulsoriamente não pode ainda ser recomendada, sendo necessários mais estudos para corroborar tal conduta. O ideal consiste na avaliação clínica pelo pediatra ou médico de família, que será capaz de investigar clinicamente as causas de cefaléia infantil, ficando ao seu critério a avaliação oftalmológica completa. A triagem visual habitual, porém, é indicada.

Vermelhidão ocular foi um sinal presente em 38 crianças, sendo notada em $11,7 \%$ das crianças com baixa acuidade visual $(p=0,87)$. No que se refere ao prurido ocular, 63 crianças queixaram-se de tal sintoma, estando este presente em $26,4 \%$ das crianças que apresentaram baixa acuidade visual $(p=0,06)$. Esses dois sintomas, geralmente associados a diversas outras doenças como o glaucoma e a conjuntivite infecciosa e alérgica, não foram relacionados à baixa acuidade visual na presente amostra. Esses devem ser avaliados cuidadosamente pelo pediatra ou médico de família quanto ao diagnóstico diferencial e à necessidade de encaminhamento ao oftalmologista, não indicando uma avaliação oftalmológica completa compulsória, já que são pouco sensíveis e específicos, devendo ser realizada a triagem visual habitual.

A pesquisa dos dois sinais e sintomas relacionados 
à baixa acuidade visual deve fazer parte da triagem visual, podendo esses ser preditores da presença de alguma doença visual infantil. É recomendada, também, a realização em todas as crianças da triagem visual habitual, conforme recomendado pela Organização Mundial de Saúde. É sugerida a realização de novos estudos sobre o assunto proposto, a fim de relacionar definitivamente esses sinais e sintomas à baixa acuidade visual, para que a triagem visual seja mais efetiva. O grupo pesquisador também acha importante salientar que pediatras e médicos de família deveriam ser capazes de realizar triagem visual, fazer o diagnóstico diferencial e a abordagem terapêutica das principais patologias oculares da infância, que, se não tratadas em tempo hábil, podem ter conseqüências importantes na vida da criança, e referir seu paciente ao oftalmologista quando necessário, não devendo manejar doenças de alto nível de complexidade se não apto para tanto.

\section{Conclusão}

No presente estudo, portanto, buscou-se correlacionar a baixa acuidade visual aos principais sinais e sintomas oculares. Observaram-se dados estatisticamente significantes na relação entre dificuldade para ver o quadro e dor ocular com a baixa acuidade visual $(\mathrm{p}<0,05) . \mathrm{Na}$ associação entre cefaléia, prurido e vermelhidão ocular com a baixa acuidade visual, não foi possível estabelecer relação estatisticamente significante $(\mathrm{p}>0,05)$.

\section{Referências}

1.Temporini ER. Ação preventiva em problemas visuais de escolares. Rev. Saúde Pública. 1984; 18(3):259-62.

2.Kara-José N, Almeida GV, Arieta CEL, Araújo JS, Becgara SJ, Oliveira PR. Causas de deficiência visual em crianças. Bol. Of. Sanit. Panam, 1994; 97(5): 405-12.

3.Kara-José N, Temporini ER. Avaliação dos Critérios de triagem visual de escolares da primeira série do primeiro grau. Rev. Saúde Pública. 1980; 14(2):205-14.

4.Sperandio AMG. Capacitação de professores e pajens para detecção precoce de problemas visuais - relato de uma experiência. Rev Bras Saúde Esc. 1990; 1(1):22-5.
5.Kara-José N, Alves MR. Problemas oculares mais freqüentes em escolares. In: Condição, JAN. editor. Saúde Escolar: A criança, a vida e a escola. São Paulo: Sarvier; 1994. p. 195-203.

6.Kara-José N, Alves MR. O que fazer pela saúde ocular de nossas crianças. In: Kara-José NR, Alves MR. autores. O olho e a visão. Petrópolis (RJ): Vozes; 1995. p. 79-84.

7.Trindade JC. Teste de triagem para avaliação das acuidades visuais e auditivas em escolares. Ped Moderna. 1991; 27(5):336-8.

8.Gasparetto MERF, Temporini ER, Carvalho KMM, Kará-José N. Dificuldade visual em escolares: conhecimentos e ações de professores do ensino fundamental que atuam com alunos que apresentam visão subnormal. Arq Bras Oftalmol. 2004; 67(1):65-71.

9.Ehrlich MI, Reinecke RD, Simons K. Preschool vision screening for amblyopia and strabismus: programs, methods, guidelines. Surv Opthalmol. 1983; 28;145-63. 10.Wasserman RC, Croft CA, Brotherton SE. Preschool vision screening in pediatric practice. A study from the pediatric research in office settings (PROS) network. Pediatrics. 1992; 89: 834-8.

11.Curnyn KM, Kaufman LM. The eye examination in the pediatrician's office. Pediatr Clin N Am. 2003; 50: 25-40. 12.Faria AMC, Dias JCS, Alves MC, Alves PC, Viana MRA. Triagem oftalmológica em crianças e adolescentes do projeto esportista cidadão/casa Menino no Parque - Belo Horizonte. In: Anais do $7^{\circ}$ encontro de extensão da Universidade Federal de Minas Gerais; 2004 Set 12-15, Belo Horizonte, Brasil; 2004. Disponível em: http:// www.ufmg.br/proex/arquivos/7Encontro/Saude190.pdf. 13.Degrazia JEC, Pellin JO, Silva RA. Saúde Escolar: levantamento das deficiências visuais mais freqüentes em população escolar de Porto Alegre. Rev Amrigs. 1986; 30(3): 231-3.

14. Carvalho CT, Carvalho DC, Colaiácovo SM, Duarte GM, Fófano R, Lima MFS, et al. Acuidade visual em escolares do ciclo básico de escolas pública e particular no município de Jundiaí. Perspectivas Médicas. 1999; 10(2): 20-22. 
15.Suzuki CK, Osawa A, Amino CJ, Yamashiba CH, Matuda E, Takei LM et al. Saúde ocular de alunos de primeira a oitava séries do primeiro grau de escolas estaduais de Säo Paulo, SP - 1992. Rev Bras Saúde Esc. 1992;2: 193-7.

16.Lopes CJA, Casella AMB, Chuí CA. Prevalência de acuidade visual reduzida nos alunos da primeira série do ensino fundamental das redes pública estadual e privada de Londrina-PR, no ano de 2000. Arq Bras Oftal. 2002; 65(6): 659-64.

17.Figueiredo RM, Santos EC, Jesus IA, Castilho RM, Santos EV. Proposição de detecção sistemática de perturbações oftalmológicas em escolares. Rev. Saúde Pública. 1993; 27(3): 204-9.

18.Hendricks TJ, DE Brabander J, van Der Horst FG, Hendrikse F, Knottnerus JA. Relationship between habitual refractive errors and headache complaints in schoolchildren. Optom Vis Sci. 2007; 84(2):137-43.

\section{Agradecimentos}

Os autores agradecem ao Dr. Luciano Guimarães Ártico pelo seu auxílio na supervisão da coleta de dados, à Ms. Maria Christine Quilfeldt Carara, pedagoga, cuja contribuição auxiliou o desenho deste estudo, e à coordenadora de desenvolvimento social da Pró-Reitoria de Extensão da Universidade de Caxias do Sul, Rosane Hambsch do Nascimento, por seu apoio ter tornado possível a implementação desta pesquisa no último ano do Projeto Saúde é Cidadania/Ação Comunitária na Universidade de Caxias do Sul.

Esta pesquisa foi realizada pelo Serviço de Oftalmologia do Projeto Saúde é Cidadania/Ação Comunitária da Universidade de Caxias do Sul e foi financiada pela PróReitoria de Extensão e Coordenadoria de Desenvolvimento Social da Universidade de Caxias do Sul.

\section{Endereço para correspondência:}

Jonathan Soldera

Rua Ernesto Casara, 1056

Bairro: Salgado Filho

CEP 95098-140

Caxias do Sul (RS)

\section{Endereço eletrônico:}

jonathansoldera@gmail.com. 